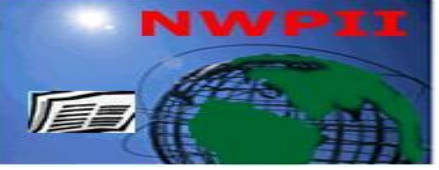

American Journal of Biomedical Sciences

ISSN: 1937-9080

nwpii.com/ajbms

\title{
Evaluation of the Ability of Short and Long Double-stranded RNAs to Induce Homologous Gene Silencing in the Protozoan Parasite, Toxoplasma gondii
}

\author{
Anna K. Crater, Ahmed Cherry, Michael Holmes, Dema Kadri, and Sirinart Ananvoranich*
}

Department of Chemistry and Biochemistry, University of Windsor, Windsor, Ontario, Canada

*Corresponding author:

Sirinart Ananvoranich, Ph.D.

Department of Chemistry and Biochemistry

University of Windsor

Windsor, Ontario N9B3P4

Canada

Phone: 519-253-3000 ext 3550

Fax: 519-973-7098

Email: anans@uwindsor.ca

Received: 1 August 2011; | Revised: 19 September 2011; | Accepted: 27 September 2011

\begin{abstract}
Among recently developed genetic tools, RNA interference (RNAi) is one of the most effective methods used in eukaryotes. Although an RNAi-like mechanism can be invoked in Toxoplasma gondii, a highly effective RNAi methodology is still elusive. Using a dual luciferase system, the ability of short (28 nucleotides) and long dsRNA ( 600 nucleotides) as RNA effectors controlling gene expression in T. gondii was evaluated. An effective down regulation can be observed when $\leq 1$ microgram of short dsRNAs were used in the down regulation of Renilla luciferase. We have detected that short dsRNAs are better gene modulators than long dsRNA. Most importantly, due to its high sensitivity and reproducibility, the dual luciferase reporter system reported here offers an excellent tool to be used in (i) the standardization of the RNAi silencing methodology performed in different Toxoplasma laboratories, (ii) the detailed mechanistic study of the RNAi pathway, and (iii) the development of better genetic tools for the biological study of protozoan parasites.
\end{abstract}

Keywords: Protozoan parasite, Toxoplasma gondii, Homologous gene silencing, Dual luciferase assays, Silencing RNAs.

\section{Introduction}

Since its discovery [1,2], RNA interference (RNAi) has become one of the most effective tools used in biological research for altering gene expression patterns for studying functional genetics. The RNAi methodology has expanded to serve many applications and purposes in both 
diagnostic and medical sciences [3,4]. Fundamentally, RNAi is a naturally occurring, evolutionary conserved, highly efficient and specific pathway by which short double-stranded RNAs (dsRNA) trigger the inhibition of gene expression [5,6]. In metazoan, dsRNAs are expressed in the form of long primary transcripts, called pri-miRNAs. Pri-miRNAs are single, foldback molecules that form dsRNA structures by intramolecular interactions, which are then processed by one or more RNase III members (i.e. Drosha and Dicer) into short and functional silencing RNAs, called miRNAs [3,7]. One single strand of the miRNA, called the mature guide or antisense strand, is then loaded onto the RNAinduced silencing complex (RISC), which allows it to bind its mRNA target to induce gene silencing [8]. In protozoan, such a mechanism has been elucidated in Trypanosoma brucei, a parasitic and pathogenic kinetoplast [9], which has a more complex silencing mechanism in Tetrahymena thermophila, a ciliated paramecium [10].

The discovery of an RNAi mechanism in $T$. brucei [9] has sparked considerable interest in exploiting the mechanism for the study of pathogenesis in a variety of pathogenic protozoan parasites, including T. cruzi (Chagas disease), Leishmania spp. (leishmaniasis), Plasmodium spp. (malaria) and T. gondii (toxoplasmosis). Limited success has been reported [11], and its possible causes could lay in their evolutionary divergence [12]. The genome sequence revealed the absence of RNAi genes in T. cruzi [13] and old-world $L$. major and L. donovani [14], thus explaining their lack of response to dsRNA. Enigmatically, $P$. falciparum, whose genome lacks conserved RNAi-genes, was shown to exhibit homologous gene silencing, akin to RNAi, where dsRNA could trigger down-regulation of gene expression [15]. Based on their evolutionary divergence, only some species have conventional RNAi genes and functions; while others retain only some homologs and/or functions. More investigations are required to unravel the mechanism of homologous gene silencing and to render a fruitful genetic tool for these protozoan parasites.

Among these evolutionarily distant protozoan organisms, T. gondii harbors homologs of Dicer,
Argonaute and RNA-dependent RNA polymerase [11,16,17]. T. gondii, an obligate intracellular parasite of the phylum Apicomplexa, is one of the most successful parasitic pathogens that has infected an estimated one third of the population and is a major threat in immunocompromised individuals [18]. To gain a better understanding of the biology and pathogenesis of $T$. gondii, a homologous gene silencing via RNAi methodology offers an excellent genetic tool. Although the canonical RNAi mechanism has not yet been demonstrated in $T$. gondii, it was shown that miRNAs in $T$. gondii (Tg-miRNAs) are expressed in a high abundance in some lineage strains such as, RH (type I), PRU (type II) and CTG (type III) [16]. Some of these annotated $T g$ miRNAs were shown to associate with translation machinery, suggesting their functions in the regulation of gene expression similar to other eukaryotes. Moreover the possible targets of $\mathrm{Tg}$ miRNA were predicted to interact by perfect or partial base-pair interaction similar to those of siRNA and miRNA of other eukaryotes [16].

Despite a lack of a mechanistic characterization, our group is one of few laboratories which successfully showed that homologous gene silencing, similar to RNAi, is functional and can be used in the study of $T$. gondii biology [19-26]. However, these results have proved difficult to reproduce in other laboratories [11]. Here, in an attempt to standardize homologous gene silencing in $T$. gondii, a dual luciferase reporter system was employed so that the expression of Photinus (firefly, Ffluc) and Renilla (Rnluc) luciferases, served as an internal control and a test target, respectively. Such a reporter system would allow any interested laboratories to set up comparable experiments to ensure reproducibility of the RNAi activity. A similar reporter system was successfully used in the study of an RNAi mechanism in mammalian cell cultures, including Hela, Cos-7 and Huh-7 cells [2,27]. The reporter system would thus allow for an unbiased analysis of the silencing capability and effectiveness found in $T$. gondii, in comparison to those of other organisms. In addition, we have demonstrated that both long and short dsRNA are capable of invoking homologous gene silencing in $T$. gondii 
with different silencing abilities. Here we referred to the short dsRNA with perfect-matched central bases as "siRNA", and that with mismatched central bases as "miRNA". This study is the first to show the ability of miRNA to induce gene silencing in $T$. gondii.

\section{Materials and Methods}

\subsection{Cell and parasite cultures}

Human foreskin fibroblasts (HFF; ATCC, \#1041) were maintained using Dulbecco's Modified Eagle Media (D-MEM) with high Dglucose and L-glutamine (Invitrogen, \#12100046) supplemented with $10 \%$ cosmic calf serum (ThermoFisher Scientific, Hylcone, \#H3008704N) and diluted $(0.5 \mathrm{x})$ Antibiotic-Antimycotic (Invitrogen, \#15240-062) at $37^{\circ} \mathrm{C}$ in $5 \% \mathrm{CO}_{2}$. $\mathrm{RH} \Delta \mathrm{HX}, T$. gondii type I strain (NIH AIDS Research and Reference Reagent Program, \#2857) was cultured in confluent HFF using Minimum Essential Media (MEM, Invitrogen, \#61100061) supplemented with $1 \%$ dialyzed fetal bovine serum (ThermoFisher Scientific, Hyclone, \#SH3007903) and diluted (0.5x) AntibioticAntimycotic.

\subsection{Reporter plasmids}

Expression plasmids, pTubRn and pTubFf, were derived from pRL-3xBulge and pFL-Con [28] and pTub_eGFP_mCherry_CAT which were gifts from W. Filipowicz (Friedrich Miescher Institute for Biomedical Research, Switzerland) and J. Murray (University of Pennsylvania, USA), respectively. In separate reactions, the DNA fragments containing the coding sequence of Rnluc \& miRNA-testing target sites and the coding sequence of Ffluc was excised from the plasmids pRL-3xBulge and pFL-Con following (i) Not I digestion, (ii) Klenow Fragment treatment, (iii) NheI digestion and (iv) gel extraction. The resulting DNA fragment containing either designed Rnluc-silencing target or Ffluc was ligated to prepared pTub_eGFP_mCherry_CAT. In the preparation of pTub_eGFP_mCherry_CAT, the coding sequence of eGFP_mCherry fusion was removed following (i) Afl II digestion, (ii) Klenow Fragment treatment, (iii) Nhe I digestion and (iv) gel extraction to give the cloning vector carrying the T. gondii-Tubulin (TgTub) promoter and the polyadenylation signal, contained within the 3 'UTR of $T$. gondii dihydrofolate reductase (TgDHFR), flanking the Rnluc or Ffluc coding sequence, and chloramphenicol acetyltransferase cassette. Resultant pTubRn and pTubFf plasmids were subjected to restriction endonuclease analyses and nucleotide sequencing reactions to confirm their identity and correct open reading frame.

\subsection{In vitro transcription of long and short dsRNA}

In vitro transcription reactions were carried out using either $2 \mu \mathrm{g}$ DNA amplicons or $2 \mu \mathrm{M}$ annealed oligonucletide primers as templates in the presence of T7 RNA polymerase ( $\sim 5$ units), 80 $\mathrm{mM}$ HEPES-KOH (pH 7.5) $24 \mathrm{mM} \mathrm{NaCl}_{2}, 2 \mathrm{mM}$ spermidine, $40 \mathrm{mM}$ DTT, $10 \mathrm{mM}$ rNTPs, and $\sim 1$ unit pyrophosphatase in $50 \mu \mathrm{L}$ reaction mixtures at $37^{\circ} \mathrm{C}$ for 16 hours, similar to previously described conditions [26]. Resultant RNAs were extracted with one volume of phenol-chloroform mixture (1:1), subsequently precipitated and quantified using a spectrophotometer (Thermo Scientific NanoDrop2000). The DNA amplicons were prepared using oligonucleotide primers containing a gene specific sequence and the sequence of the T7 promoter, as listed in Table 1, under standard conditions for Taq DNA polymerase (New England Biolabs). For RnlucdsRNAs, pTubRnluc was used as templates for PCR amplification using two sets of oligonucleotide primers: FW_RnLuc_1 and RV_RnLuc_1 to yield Rnluc-dsRNA_1, or FW_RnLuc_2 and RV-RnLuc_2, to yield RnlucdsRNA_2. The amplicon used in the generation of control dsRNA was amplified from a plasmid containing $T$. gondii uracil phosphoribosyl transferase (TgUPRT) and a pair of oligonucleotide primers, called FW_UP and RV_UP.

For short dsRNA in vitro transcription, annealed oligonucleotide primers were used as templates. The oligonucleotide primers were designed to contain a complementary sequence of the T7 RNA polymerase promoter and the target sequences to independently generate sense and antisense strands of the RNA. The templates for 
the sense RNA strand synthesis were formed by heating a mixture of $2 \mu \mathrm{M}$ T7promoterGG and Sense_Tpl for 5 minutes at $70{ }^{\circ} \mathrm{C}$ and slowly cooling to room temperature. The mixture was then used in the in vitro transcription reaction. Similar procedures were carried out to create the templates for the antisense strands carrying a perfect or mismatch complementarity by using T7promoterGG to anneal with Antisense_match_Tpl or Antisense_mismatch_Tpl respectively. Following the in vitro transcription, resultant RNAs were quantified. Equal amounts of sense and antisense RNAs were mixed and annealed by heating the mixture to $70^{\circ} \mathrm{C}$ for 5 minutes and cooled to room temperature to generate double-stranded silencing RNA with perfect-matched central bases (siRNA) or with mismatched central bases (miRNA) (Figure 1). The sense and antisense strands of the control RNA were generated similarly using T7promoterGG to anneal with Sense_CTRL or Antisense_CTRL, respectively, to create templates for in vitro transcription reactions.

Table 1 Oligonucleotide primers used in the study.

The sequence of T7 RNA polymerase promoter and its complementary bases are in bold and underlined, respectively.

\begin{tabular}{|c|c|}
\hline Names & Nucleotide sequences \\
\hline FW_RnLuc_1 & TAATACGACTCACTATAGGTGTACGACCCCGAGCAAC \\
\hline RV_RnLuc_1 & TAATACGACTCACTATAGGTAGGCAGCGAACTCCTCA \\
\hline FW_RnLuc_2 & TAATACGACTCACTATAGGGACGAGTGGCCTGACATC \\
\hline RV-RnLuc_2 & TAATACGACTCACTATAGGGCAGCTTCTGTTTACTTA \\
\hline FW_UP & TAATACGACTCACTATAGGTTCATGAGCACCACATTGGG \\
\hline RV_UP & TAATACGACTCACTATAGGGTTCCAAAGTACCGGT \\
\hline T7promoterGG & TAATACGACTCACTATAGG \\
\hline Sense_Tpl & AATGAGGTAGTTCAATAGGCTGTGCCTATATGAGTCGTATTA \\
\hline Antisense_match_Tpl & AACACAGCCTATTGAACTACCTCACCTATATGAGTCGTATTA \\
\hline Antisense_mismatch_Tpl & AACACAGCCTACCTGGCTACCTCACCTATATGAGTCGTATTA \\
\hline Sense_CTRL & AAGGGATCGACAAGGAGCTTTCCTATAGTGAGTCGTATTAC \\
\hline Antisense_CTRL & AAGGAAAGCTCCTTGTCGATCCCTATAGTGAGTCGTATTAC \\
\hline siRNA & $\begin{array}{ll}\text { 5' aaugagguaguucaauaggcugugccuu } & 3 \text {, sense strand } \\
\text { 3' unccacuccaucaa guuauccgacacaa } & 5^{\prime} \text {, antisense strand }\end{array}$ \\
\hline miRNA & 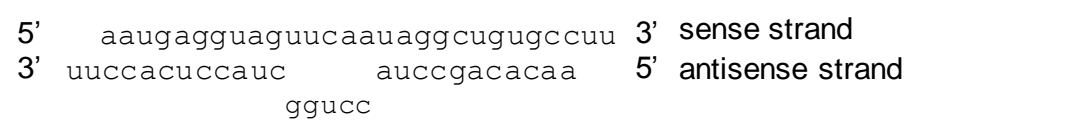 \\
\hline
\end{tabular}

Figure 1. Sequences of short dsRNAs. 
A.

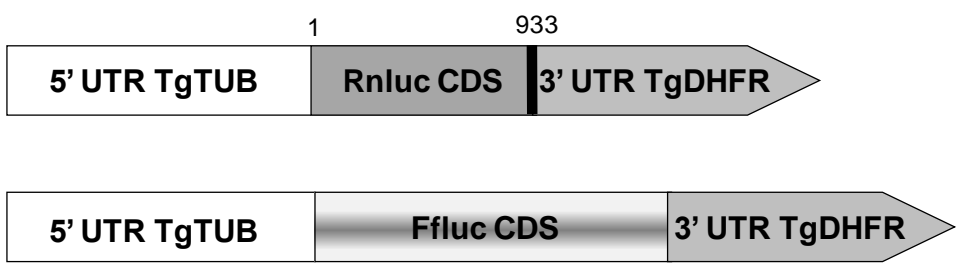

B.

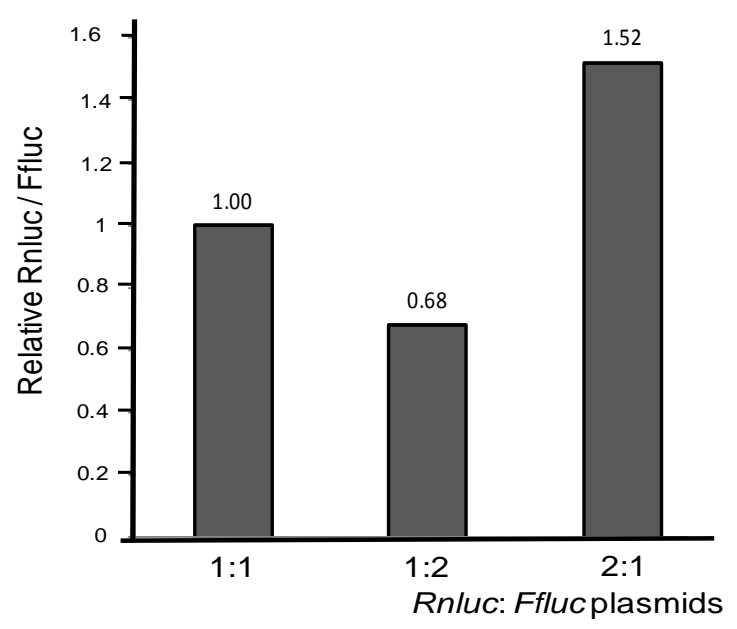

C.

\begin{tabular}{ccc}
\hline $\begin{array}{c}\text { Rnluc/Ffluc } \\
\text { Total amount }(\boldsymbol{\mu g})\end{array}$ & $\begin{array}{c}\text { Rnluc activity } \\
\text { (light units) }\end{array}$ & $\begin{array}{c}\text { Ffluc activity } \\
\text { (light units) }\end{array}$ \\
\hline 0.2 & $1974 \pm 7.1$ & $2851 \pm 3.2$ \\
2 & $2972 \pm 5.8$ & $3975 \pm 2.1$ \\
20 & $4434 \pm 10.2$ & $10319 \pm 4.8$ \\
40 & $34086 \pm 5.4$ & $38675 \pm 4.4$ \\
& $41980 \pm 3.9$ & $50044 \pm 3.6$ \\
\hline
\end{tabular}

Figure 2. Dual luciferase reporter system. (A) Schematic representation of mRNA reporters used in this study. Numbers indicate the first nucleotide of the start codon and the last nucleotide of coding sequence. The transcript encoding Rnluc was used as target for the gene silencing study and from which silencing RNAs were designed (see Table 2 and Materials and Methods). (B) Dual luciferase expression was assayed using different ratios of Rnluc and Ffluc plasmids, while the total amount of plasmids were kept at $60 \mu \mathrm{g}$ for the transfection. The graph is a representative of compiled experimental results. (C) Dose-response of dual luciferase expression was assessed when the ratio of Rnluc:Ffluc plasmids was kept at 1:1 ratio. The expression of the dual luciferase is shown as relative Rnluc/Fluc light forming units. The assays were carried out 24 hours post-electroporation. Values were obtained from at least two independent experiments. 


\subsection{Dual luciferase assay following transfection by electroporation}

Freshly lysed parasites were harvested and counted. Approximately $2 \times 10^{6}$ parasites were used for each transfection via electroporation using a BTX ECM 630 (1500 volts, $25 \Omega$, and 25 $\mu \mathrm{F})$. For each transfection, reporter plasmids with or without tested RNAs were mixed with harvested parasites in an electroporation mixture $(400 \mu \mathrm{L})$ containing $120 \mathrm{mM} \mathrm{KCl}, 0.15 \mathrm{mM}$ $\mathrm{CaCl}_{2}, 10 \mathrm{mM} \mathrm{K} \mathrm{HPO}_{4} / \mathrm{KH}_{2} \mathrm{PO}_{4}(\mathrm{pH}$ 7.6) $2 \mathrm{mM}$ EDTA, $5 \mathrm{mM} \mathrm{MgCl}_{2}, 2 \mathrm{mM}$ ATP, $5 \mathrm{mM}$ glutathione in a $4 \mathrm{~mm}$-gap cuvette and electroporated [29]. Following electroporation, the parasites were cultured in confluent HFF monolayers grown in T25 flasks using MEM media with L-glutamine without phenol red (Sigma Aldrich, \#M3024) and supplemented with $1 \%$ dialyzed fetal bovine serum and diluted $(0.5 \mathrm{x})$ Antibiotic-Antimycotic at $37^{\circ} \mathrm{C}$ in $5 \% \mathrm{CO}_{2}$ for selected periods.

For dual luciferase assays, the infected monolayers were harvested and lysed with $100 \mu \mathrm{L}$ of 1x Passive Lysis Buffer (Promega, \#E1531) and incubated for 10 minutes at room temperature. Lysates were cleared of debris by centrifugation, and the supernatant was kept on ice and used in the dual luciferase assay, which was carried out in a 2-step fashion. For a Ffluc assay, $20 \mu \mathrm{L}$ of lysate was added to a freshly made reaction mixture (100 $\mu \mathrm{L}$ ) containing $200 \mu \mathrm{M}$ D-luciferin (Sigma Aldrich, \#L9504), $20 \mathrm{mM}$ Tricine, $10 \mathrm{mM}$ $\mathrm{MgSO}_{4}, 5 \mathrm{mM}$ DTT, $250 \mu \mathrm{M}$ ATP and $250 \mu \mathrm{M}$ Coenzyme A. The mixture was incubated for 10 seconds at room temperature. For a Rnluc assay, $20 \mu \mathrm{L}$ of lysate was added to a freshly made reaction mixture $(100 \mu \mathrm{L})$ containing $0.1 \mu \mathrm{M}$ Coelenterazine (Santa Cruz Biotechnology Inc, \#sc-205908), $100 \mathrm{mM} \mathrm{K} 2 \mathrm{HPO}_{4} / \mathrm{KH}_{2} \mathrm{PO}_{4}$ (pH 7.6) $500 \mathrm{mM} \mathrm{NaCl}, 1 \mathrm{mM}$ EDTA, and $0.02 \%$ BSA. The mixture was incubated for 10 seconds at room temperature. The luminescence signals from both reactions were measured with a $20 / 20^{\mathrm{n}}$ Luminometer (Turner Biosystems). Rnluc signals were normalized to Ffluc signals for a direct comparison among different independent experiments. For each experiment, the reporter plasmid electroporation without tested RNA, called mock electroporation, was carried out to allow for the measurement of the dual luciferase expression under independently tested conditions. At least two independent experiments were performed.

\section{Results and Discussion}

\subsection{Strategy \& dual luciferase reporter system}

In the design of the dual luciferase reporter system for the gene silencing study, we aimed to have an equal transcript production of both transcription units. Therefore, the reporter system was engineered so that the expression of both Ffluc and Rnluc are under the control of a strong and constitutive $T$. gondii promoter, called TgTubulin, which also provides the necessary 5'UTR for the protein's expression. Furthermore, to ensure similar levels of transcript stability and protein synthesis from both constructs, the 3'UTR of TgDHFR (dihydrofolate reductase) were placed downstream from the coding sequence (Fig. 2A). The transcript derived from Rnluc was used as the target for the homologous gene silencing by three different RNA modulators, including long dsRNA, miRNA and siRNA, while the Ffluc transcript served as an internal control for transfection, expression, and detection.

Due to the parasitic nature of $T$. gondii, in a gene expression and silencing analysis, the reporter system and silencing RNAs would be introduced into the parasite prior to infection of a host monolayer. We viewed that the success of the subsequent analysis would rely greatly on the viability of the post-electroporated parasite and the efficiency of transfection. The viability of the post-electroporated parasite was evaluated using the ability of the viable parasite to exclude trypan blue and propidium idodide [30]. From three independent experiments, we detected that $75 \pm$ $10 \%$ were viable and able to multiply, when up to $60 \mu \mathrm{g}$ of nucleic acids (RNA or DNA) were used in the elctroporation conditions (see Material and Methods). The efficiency of transfection was evaluated by determining the amounts of remaining and internalized nucleic acids following electroporation. Nucleic acids (RNA or DNA) were randomly labeled with fluorescein during in 
vitro transcription or PCR amplification, and used in the electroporation. Approximately 52\% of labeled nucleic acids were internalized (Al-Anouti \& Ananvoranich, unpublished data). We thus concluded that under similar controlled conditions, the electroporation of nucleic acids $(\leq 60 \mu \mathrm{g}$, DNA plasmids and/or RNAs) would allow sufficient numbers of viable parasites for gene silencing assays.

Next we assessed the expression of our newly engineered dual luciferase system to allow for a sensitive detection of a broad range of different silencing activities, while remaining within a linear range for a direct comparison. Based on a previous report [31] using a similar reporter system in $T$. gondii, when a promoter of interest and a well-characterized promoter were placed to control the expression of Rnluc and Ffluc, respectively, the transfection was carried out using $60 \mu \mathrm{g}$ of the Rnluc and Ffluc reporter plasmids (1:2 ratio). Keeping the total amount of DNA used at $60 \mu \mathrm{g}$, we varied the ratio of Rnluc:Ffluc at either $1: 1,1: 2$ or $2: 1$. All ratios tested yielded reproducible data (Fig. 2B). Consequently, the plasmid ratio at 1:1 was kept for simplicity. Other experimental parameters, including the total plasmid concentration, the number of extracellular parasites, and incubation time (post-transfection and luciferase assays), were evaluated in different combinations to establish optimal conditions for the study.

When varied numbers of harvested parasites were used in the transfection, $\sim 10^{5}-10^{6}$ freshly released parasites were the most suitable for the study because the parasites remained intracellular at 24,48 or 72 hours post-electroporation under the conditions tested (see Materials and Methods). These data indicated that both transcription units were efficiently expressed and maintained during the testing period ( $\leq 3$ days). Because lower amounts of the reporter system were used in other eukaryotic systems [2,27], we thus progressively lowered the total amounts of plasmids from 40 to $0.2 \mu \mathrm{g}$ (Ffluc:Rnluc $=1: 1$ ) so that the gene silencing data could be directly compared. It was detected that within the amounts of plasmids tested; both transcription units were still sufficiently expressed and allowed for detection within a linear range (Fig. 2C). In all succeeding silencing analyses, $1 \mu \mathrm{g}$ of each reporter plasmid ( $2 \mu \mathrm{g}$ total) was used.

\subsection{Homologous gene silencing by long double- stranded RNA}

Long dsRNAs (ca. 300-800 nts) have previously been shown to induce homologous gene silencing in $T$. gondii [22-26]. To test our newly developed reporter system, two species of long dsRNAs were designed to have homologous sequences to the Rnluc mRNA. Rnluc-dsRNA_1 (596 nts) was homologous to nucleotides 12 to 607 of the Rnluc coding sequence, and RnlucdsRNA_2 (589 nts) was derived from the Rnluc coding sequence at nucleotides $458-933$ and the

Table 2 List of silencing RNAs used in the study.

Numbers of the nucleotides are corresponding to the Rnluc transcript depicted in Figure 2A.

\begin{tabular}{ccc}
\hline Silencing RNAs & $\begin{array}{c}\text { Homologous to } \\
\text { regions }\end{array}$ & $\begin{array}{c}\text { Homologous to Rnluc transcript } \\
\text { (Nucleotides) }\end{array}$ \\
\hline Rnluc dsRNA_1 & CDS & $12-607$ \\
Rnluc dsRNA_2 & CDS \& 3'-UTR & $458-1045$ \\
siRNA & 3'-UTR & $943-965,972-994,1000-1022$ \\
miRNA & 3'-UTR & $943-965,972-994,1000-1022$ \\
\hline
\end{tabular}


proximal region (113 nts) of its 3'-UTR (Table 2). These long dsRNAs have an overlapping region (149 nts) spanning the nucleotides 458 to 607 of the Rnluc coding sequence. Using these dsRNAs, we aimed to evaluate whether dsRNAs derived from these regions exhibited similar silencing inducing ability. This in turn would reflect intermolecular interactions formed between the mRNA target and different dsRNAs, given potential structural preferences.

To determine the extent of dsRNA ability to induce gene silencing, varied amounts of tested dsRNAs $(0.25$ to $4 \mu \mathrm{g})$ were co-transfected along with $2 \mu \mathrm{g}$ reporter plasmids (1:1) by electroporation. When $0.25 \mu \mathrm{g}$ dsRNA was used, a negligible reduction $(<10 \%)$ of the relative Rnluc/Ffluc level was detected at 24 hours postelectroporation (Fig. 3A). A clear silencing effect $(\sim 30-40 \%)$ was observed, when $0.5 \mu \mathrm{g}$ of RnlucdsRNA_1 and 2 were used and able to reduce the relative Rnluc/Ffluc level to $73 \pm 4 \%$ and $61 \pm$ $1 \%$, in comparison with a mock silencing control (without dsRNA). The silencing effects were slightly increased when higher amounts of both dsRNAs were used. The maximal silencing ability was observed when 2-4 $\mu \mathrm{g}$ dsRNA caused the relative Rnluc/Ffluc reduction to $52 \pm 10 \%$ and 62 $\pm 1 \%$ for Rnluc-dsRNA_1 and dsRNA_2, respectively at 24 hours post-electroporation (Fig. 2A). Similar levels of silencing were observed when the electroporated parasites were allowed to incubate longer, and the dual luciferase assays were carried out at 48 hours post-electroporation (Supplementary Figure 1). The findings suggested that the transfection of long dsRNAs at $2 \mu \mathrm{g}$ or $\sim 5.1$ pmoles, despite their targeted regions, have saturated the silencing machinery and reached their maximal ability to cause a silencing effect under conditions tested.

To evaluate the specificity of the homologous gene silencing, an unrelated dsRNA, whose nucleotide sequence is different from the intended target (the Rnluc transgene) was tested under similar conditions. TgUPRT dsRNA (629 nts) did not cause any silencing effect on Rnluc expression (Fig. 3A, the lightest shaded bars), indicating that the silencing effect is sequence specific. The presence of unrelated dsRNA (as high as $4 \mu \mathrm{g}$ or 10.3 pmoles) did not affect the activity of Rnluc or
Ffluc. It thus indicated that in a unicellular organism, such as $T$. gondii, the presence of long dsRNA in a large quantity $(\leq 4 \mu \mathrm{g})$ did not cause a general cellular response affecting the transcription processes and/or the degradation of gene products over the tested conditions.

In summary, we have confirmed that a homologous long dsRNA has the ability to induce gene silencing in $T$. gondii. DsRNA exhibited the ability to induce Rnluc silencing and to drop the relative Rnluc/Ffluc to $60 \%$ in comparison to a mock electroporation. This silencing effect ( $40 \%$ reduction of gene expression) was considered a mild effect in comparison to when miRNA was used in silencing ( $\sim 80 \%$ reduction) the expression of Rnluc in mammalian cell cultures [2,27]. However, such a mild silencing ability could be useful, when it is sufficient to produce a detectable phenotype (i.e. the change in an enzymatic activity). However, when TgUPRT or Toxoplasma lactate dehydrogenases (TgLDHs) were silencing targets, their homologous dsRNAs were able to reduce their expression by $\sim 90 \%$. In those experiments, we detected the silencing outcomes by radioactive uracil uptake assays for TgUPRT study [25] and by in vitro and in vivo growth and differentiation assays for TgLDH1 and TgLDH2 experiments [24]. It is highly likely that the level or extent of silencing effect is gene-dependent, since here the silencing effect towards Rnluc was observed at $\sim 40 \%$, similar to that of Toxoplasma enolase experiments [22].

Despite its mild silencing ability, a homologous dsRNA-induced gene silencing procedure offers a very easy-to-use method. One would require little information about the gene target, such as the sequence of the transcripts or the exons, prior to initiating a silencing protocol. Moreover, long dsRNAs can quickly be synthesized in a very cost-effective way via in vitro transcription. One drawback of the system would be due to its inherited long target region(s). When more than one species of transcripts are produced from the same exon(s), the long homologous dsRNA would not be able to discern one transcript over another. Considering its pros and cons, this homologous dsRNA-induced gene silencing methodology is a valuable method-ofchoice. Keeping in mind, with its potentially mild 
silencing ability, the lack of a desirable phenotype might not indicate the failure of the silencing procedure, but could be an indication of the critical level of gene expression required for maintaining a phenotype.

A.

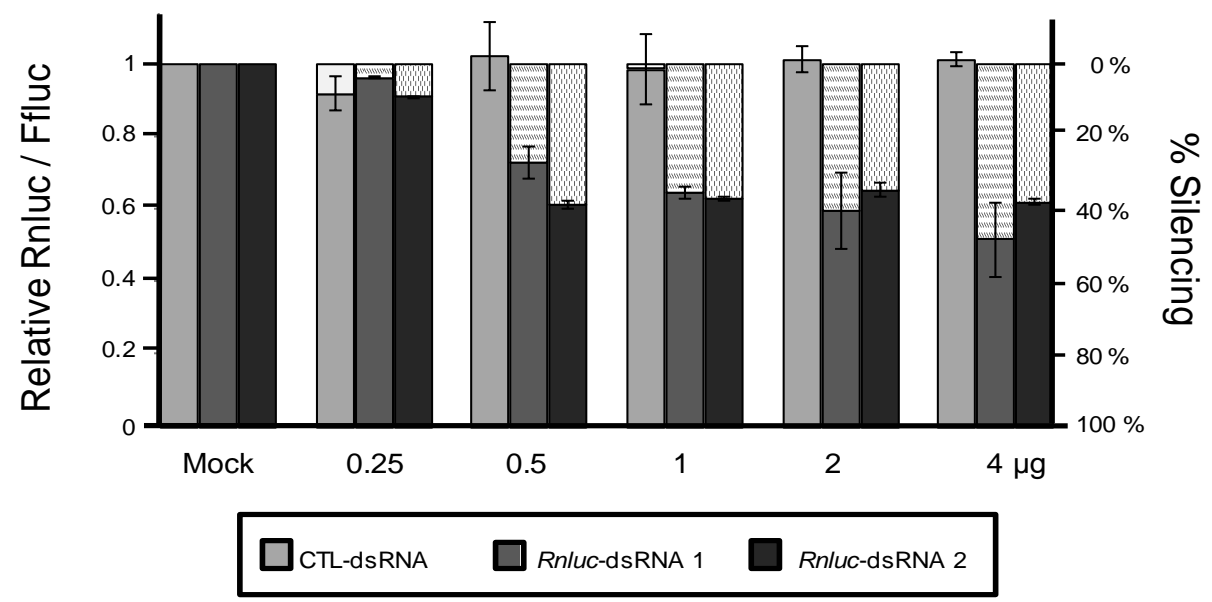

B.

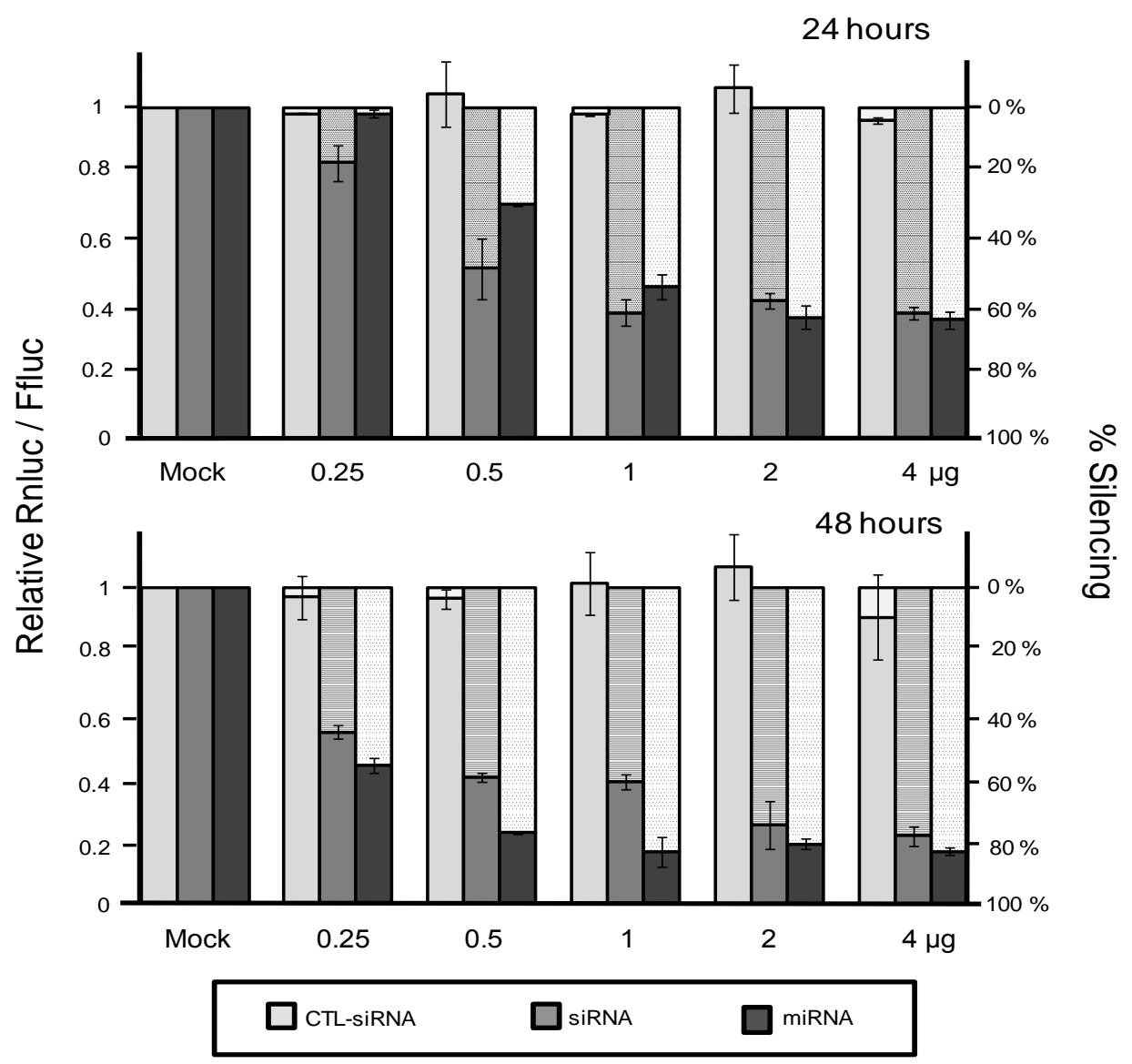

Figure 3. Gene silencing effect caused by (A) long dsRNA was detected at 24 hours post-electroporation (B) and siRNA and miRNA at 24 hours and 48 hours post-electroporation. Mock electroporations were performed using only the reporter plasmids ( $1 \mu \mathrm{g}$ of each Rnluc and Ffluc plasmid) in the absence of tested silencing RNAs. 


\subsection{Homologous gene silencing by short double- stranded RNAs}

Due to their availability and reliability to induce gene silencing, gene-specific siRNAs and miRNAs have become powerful reagents used in biological research. Recent genomic and biochemical research has pointed that the $T$. gondii genome harbors both the RNAi genes and miRNAs [11,16,17]. It was thus crucial to investigate whether difference in complementarity could induce gene silencing of designated gene target. Based on predicted secondary structures and known constraints of short dsRNAs [32], we used the mfold nucleic acid folding and hybridization prediction software [33] and SciTool RNAi design software (Integrated DNA Technologies, Inc., Iowa) to predict the most probable target sites and to design short dsRNAs specific to the Rnluc transcripts. Twenty possible targets and siRNA candidates were predicted and ranked from the most to the least probable location for the interactions. We chose the three most probable sites located between the Rnluc stop codon and the proximal region (103 nts) of its 3'UTR (Table 2). Two silencing RNAs were designed and synthesized for the study. One contains nucleotides perfectly complementary to its target, representing a siRNA interaction; while the other, with five-central nucleotide mismatch, represents a miRNA interaction (Fig. 1). These silencing RNAs have three possible recognition sites located within the 3' UTR between the nucleotides 943 and 1022 of the Rnluc transcript (Table 2).

Varied amounts of siRNA and miRNA $(0.25$ to $4 \mu \mathrm{g}$, or 15 to 246 pmoles) were independently tested for their ability to induce gene silencing using conditions similar to those tested for the long dsRNA-induced gene silencing. At 0.25 or $0.5 \mu \mathrm{g}$, siRNA was better than long dsRNA or miRNA (at the same amounts) to lower the Rnluc expression (Fig. 3B, upper panel) at 24 hours postelectroporation. Both siRNA and miRNA at 1 to 4 $\mu \mathrm{g}$ exhibited similar ability to silence the expression of Rnluc ( $\sim 60 \%$ reduction). Interestingly, when the silencing assays were carried out at 48 hours post-electroporation, the effect was more pronounced for all amounts of
siRNA and miRNA tested (Fig. 3B, lower panel). Both siRNA and miRNA (at $0.25 \mu \mathrm{g}$ ) exhibited similar silencing ability (54 $\pm 2 \%$ reduction), which was higher than those at 24 hours. At higher amounts used, miRNA seems to show slightly better ability than siRNA to induce gene silencing. This silencing effect was not detected with an unrelated small RNA at either 24 or 48 hours (Fig. 3B, lightly shade bars), confirming the homologous nucleotide sequence was required.

An enhanced silencing effect found at a longer incubation was not observed in dsRNAinduced gene silencing (Fig. S1). It is possible that siRNA- or miRNA-engaged machinery could be more stable than, or different from, that of a dsRNA-system. Considering the nature of transient expression of the reporter plasmids, the more stable the silencing machinery is, the more robust the machinery is to compete against the expression of reporters. Another possibility could be due to the presence of an RNA-dependent RNA polymerase, to which $T$. gondii carries a homologue, which would allow for the amplification of short dsRNAs using the transcripts as templates. Thus, their silencing ability would remain longer and could be enhanced as long as the transcript is present.

In summary, our findings clearly indicated that small silencing RNAs (siRNA and miRNA) are able to specifically induce gene silencing in $T$. gondii. This study is the first to demonstrate the activity of miRNA in gene silencing in the Apicomplexan protozoan parasites. More importantly, we showed that the silencing effect was within the range ( $\sim 80 \%$ reduction of gene expression) of those observed in the silencing study in mammalian cell cultures [2,27]. Due to their short recognition sequence, both siRNAs and miRNAs would interact with their target more specifically and yield less potential off-target interactions than long dsRNA.

\subsection{Which silencing RNA (long dsRNA, siRNA and miRNA) is the best to induce homologous gene silencing?}

Using the dual luciferase reporter system, we demonstrated that silencing RNAs, including long dsRNA, siRNA (perfect-match bases) and miRNA 
(central-mismatch bases), were capable of inducing gene-specific silencing. Long dsRNA exhibited the least silencing effect ( 40\% reduction of gene expression). The mild silencing effect was maintained for 48 hours, which could be due to (i) additional processing steps required to process the long dsRNA into small silencing RNAs, or (ii) a different silencing mechanism being engaged. As stated earlier, long dsRNAinduced gene silencing could be a useful tool when a quick assessment is required for evaluating a putatively essential gene. In a shorter tested period (24 hours), siRNA and miRNA were equally effective where the silencing effect was $\sim 50 \%$. When a prolonged and high silencing effect is required, miRNA offers the best tool among these tested silencing RNAs. Tested miRNA affected the Rnluc expression the most (80\% reduction), which is equivalent to those tested in different mammalian cell cultures [2,27]. Therefore, we concluded that miRNA, with central-mismatch bases, is the best silencing RNA to use when a homologous gene silencing is required for the study of a gene of interest.

\section{Acknowledgements}

This work was supported by the Natural Sciences and Engineering Research Council of Canada (NSERC, Canada, S.A.), and the Ministry of Colleges and Universities of Ontario (M.H.).

\section{References}

1. Fire, A.; Xu, S.; Montgomery, M. K.; Kostas, S. A.; Driver, S. E.; Mello, C. C. Potent and specific genetic interference by doublestranded RNA in Caenorhabditis elegans. Nature, $\quad$ 1998, 391, 806-811. doi: $10.1038 / 35888$

2. Elbashir, S.M.; Harborth, J.; Lendeckel, J.W.; Yalcin, A.; Weber, K.; Tuschl, T. Duplexes of 21-nucleotide RNAs mediate RNA interference in cultured mammalian cell. Nature, $\quad 2001, \quad 411, \quad 494-498$. doi:10.1038/35078107

3. Grimm, D. Small silencing RNAs: state-ofthe-art. Advanced Drug Delivery Review,
2009, 61(9),

672-703.

doi:10.1016/j.addr.2009.05.002

4. Kim, D.; Rossi, J. RNAi mechanisms and applications. Biotechniques, 2008, 44(5), 613616. doi: 10.2144/000112792

5. Meister, G.; Tuschl, T. Mechanisms of gene silencing by double-stranded RNA, Nature, 2004, 431, 343-349. doi:10.1038/nature02873

6. Dykxhoorn, D.M.; Lieberman, J. The silent revolution: RNA interference as basic biology, research tool, and therapeutic, Annual Review of Medicine. 2005, 56, 401-423. doi: 10.1146/annurev.med.56.082103.104606

7. Carthew, R.W.; Sontheimer,E.J. Origins and mechanisms of miRNAs and siRNAs, Cell, 2009, 136, 642-655. doi: 10.1016/j.cell.2009.01.035

8. Brodersen, P.; Voinnet, O. Revisiting the principles of microRNA target recognition and mode of action, Nature Reviews Molecular Cell Biology, 2009, 10, 141-148. doi:10.1038/nrm2619

9. Ngo, H.; Tschudi,C.; Gull, K.; Ullu, E. Double-stranded RNA induces mRNA degradation in Trypanosoma brucei. Proceeding of the National Academy of Sciences of USA, 1998, 95, 14687-14692.

10. Schramke, V.; Allshire, R. Those interfering little RNAs! Silencing and eliminating chromatin. Current Opinion in Genetics \& Development, 2004, 14(2), 174-180. doi:10.1016/j.gde.2004.02.006

11. Kolev, N.G.; Tschudi, C.; Ullu, E. RNA Interference in Protozoan Parasites: Achievements and Challenges. Eukaryotic Cell, 2011, Epub ahead of print, Jul 15. doi:10.1128/EC.05114-11

12. Ullu, E.; Tschudi, C.; Chakraborty, T. RNA interference in protozoan parasites. Cellular Microbiology, 2004, 6(6), 509-519. DOI: 10.1111/j.1462-5822.2004.00399.x

13. El-Sayed, N. M.; Myler, P. J.; Bartholomeu, D. C.; Nilsson, D.; Aggarwal, G.; Tran, A. N.; et al. The genome sequence of Trypanosoma cruzi, etiologic agent of Chagas disease. Science, 2005, 309, 409-415. DOI: 10.1126/science. 1112631

14. Ivens, A. C.; Peacock, C. S.; Worthey, E. A.; Murphy, L.; Aggarwal, G.; Berriman, M.; et 
al. The genome of the kinetoplastid parasite, Leishmania major. Science, 2005, 309, 436442. doi: $10.1126 /$ science. 1112680

15. McRobert, L.; McConkey, G. A. RNA interference (RNAi) inhibits growth of Plasmodium falciparum. Molecular and Biochemical Parasitology, 2002, 119, 273 278. doi:10.1016/S0166-6851(01)00429-7

16. Braun, L.; Cannella, D.; Ortet, P.; Barakat, M.; Sautel, C.F.; Kieffer, S.; Garin, J.; Bastien, O.; Voinnet, O.; Hakimi, M.A. A complex small RNA repertoire is generated by a plant/fungallike machinery and effected by a metazoanlike Argonaute in the single-cell human parasite Toxoplasma gondii. PLoS Pathogens, 2010, 6(5), e1000920. doi: 10.1371/journal.ppat.1000920

17. Al Riyahi, A.; Al-Anouti, F.; Al-Rayes, M.; Ananvoranich, S. Single argonaute protein from Toxoplasma gondii is involved in the double-stranded RNA induced gene silencing. International Journal for Parasitology, 2006, 36(9), 1003-1014. doi:10.1016/j.ijpara.2006.04.014

18. Montoya, J.G.; Liesenfeld, O. Toxoplasmosis. Lancet, 2004, 363(9425), 1965-1976. doi:10.1016/S0140-6736(04)16412-X

19. Yu, L.; Gao, Y.F.; Qiao, Z.P.; Li, C.L.; Li, X.; Shen, J.L. Toxoplasma gondii: siRNA can mediate the suppression of adenosine kinase expression. Experimental Parasitology. 2008, 118(1), 96-102. doi:10.1016/j.exppara.2007.07.007

20. Yu, L.; Gao, Y.F.; Li, X.; Qiao, Z.P.; Shen, J.L. Double-stranded RNA specific to adenosine kinase and hypoxanthine-xanthineguanine-phosphoribosyltransferase retards growth of Toxoplasma gondii. Parasitology Research, 2009, 104(2), 377-383. doi: 10.1007/s00436-008-1208-9

21. Adams, B.; Musiyenko, A.; Kumar, R.; Barik, S. A novel class of dual-family immunophilins. Journal of Biological Chemistry. 2005, 280(26), 24308-24314. doi: 10.1074/jbc.M500990200

22. Holmes, M.; Liwak, U.; Pricop, I.; Wang, X.; Tomavo, S.; Ananvoranich, S. Silencing of tachyzoite enolase 2 alters nuclear targeting of bradyzoite enolase 1 in Toxoplasma gondii.
Microbes and Infection. 2010, 12(1), 19-27. doi:10.1016/j.micinf.2009.09.010

23. Ananvoranich, S.; Al Rayes, M.; Al Riyahi, A.; Wang, X. RNA silencing of glycolysis pathway in Toxoplasma gondii. Journal of Eukaryotic Microbiology, 2006, 53(1), S162163. doi: 10.1111/j.1550-7408.2006.00216.x

24. Al-Anouti, F.; Tomavo, S.; Parmley, S.; Ananvoranich, S. The expression of lactate dehydrogenase is important for the cell cycle of Toxoplasma gondii. Journal of Biological Chemistry. 2004, 279(50), 52300-52311. doi: 10.1074/jbc.M409175200

25. Al-Anouti, F.; Quach, T.; Ananvoranich, S. Double-stranded RNA can mediate the suppression of uracil phosphoribosyltransferase expression in Toxoplasma gondii. Biochemical and Biophysical Research Communications. 2003, 302(2), 316-323. doi:10.1016/S0006291X(03)00172-4

26. Al-Anouti, F.; Ananvoranich, S. Comparative analysis of antisense RNA, double-stranded RNA, and delta ribozyme-mediated gene regulation in Toxoplasma gondii. Antisense and Nucleic Acid Drug Development. 2002, 12(4), 275-281. doi:10.1089/108729002320351593.

27. Choe, J.; Cho, H.; Lee, H.C.; Kim, Y.K. MicroRNA/Argonaute 2 regulates nonsensemediated messenger RNA decay. EMBO reports, 2010, 11, 380-386. doi: 10.1038/embor.2010.44

28. Pillai, R.S.; Bhattacharyya, S.N.; Artus, C.G.; Zoller, T.; Cougot, N.; Basyuk, E.; Bertrand, E.; Filipowicz, W. Inhibition of translational initiation by Let-7 MicroRNA in human cells. Science. 2005, 309(5740), 1573-1576. doi: 10.1126/science.1115079

29. Roos, D.S.; Donald, R.G.; Morrissette, N.S.; Moulton, A.L. Molecular tools for genetic dissection of the protozoan parasite Toxoplasma gondii. Methods in Cell Biology. 1994, 45, 27-63.

30. Jones, K.H.; Senft, J.A. An improved method to determine cell viability by simultaneous staining with fluorescein diacetate-propidium iodide. Journal of Histochemistry and 
Cytochemistry. 1985, 33(1), 77-79. doi: 10.1177/33.1.2578146

31. Behnke, M.S.; Radke, J.B.; Smith, A.T.; Sullivan, W.J.; White, M.W. The transcription of bradyzoite genes in Toxoplasma gondii is controlled by autonomous promoter elements. Mol. Microbiol. 2008, 68(6), 1502-18. doi: 10.1111/j.1365-2958.2008.06249.x

32. Kim, D.H.; Behlke, M.A.; Rose, S.D.; Chang, M.S.; Choi, S.; Rossi, J.J. Synthetic dsRNA
Dicer substrates enhance RNAi potency and efficacy. Nature Biotechnology, 2005, 23, 222-226. doi:10.1038/nbt1051

33. Zuker, M. Mfold web server for nucleic acid folding and hybridization prediction. Nucleic Acids Research. 2003, 31(13), 3406-3415. doi: $10.1093 / \mathrm{nar} / \mathrm{gkg} 595$

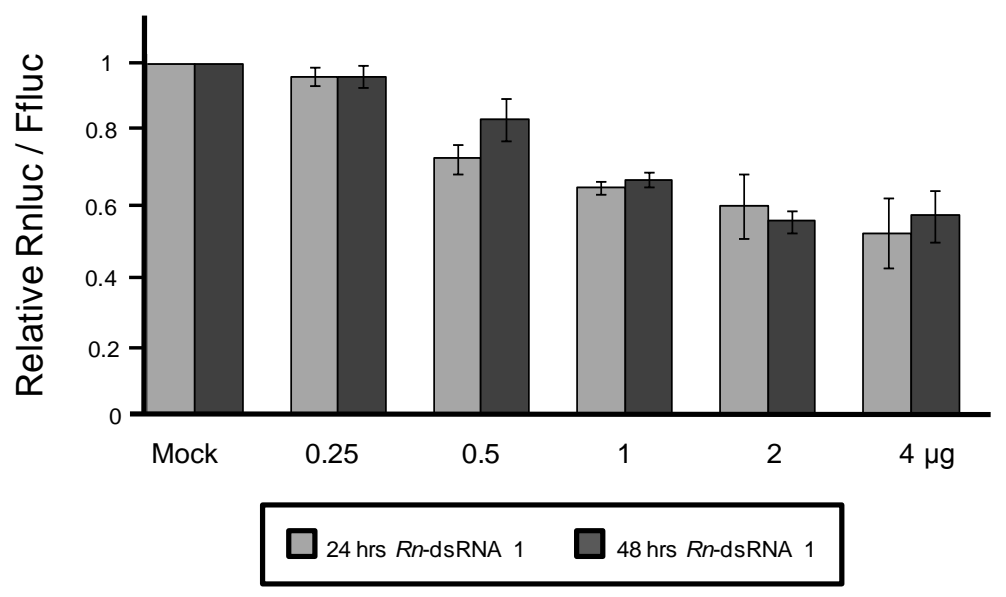

Supplementary Figure 1. Gene silencing effect caused by Rnluc-dsRNA_1 was detected at 24 and 48 hours post-electroporation. 